\title{
Ragweed pollen collected along high-traffic roads shows a higher allergenicity than pollen sampled in vegetated areas
}

\author{
A. Ghiani ${ }^{1, *}$, R. Aina ${ }^{1, *}$, R. Asero ${ }^{2}$, E. Bellotto ${ }^{1}$ \& S. Citterio ${ }^{1}$ \\ ${ }^{1}$ Dipartimento di Science Ambientali, Università di Milano-Bicocca, Milano, Italy; ${ }^{2}$ Ambulatorio di Allergologia, Clinica San Carlo, Paderno \\ Dugnano (MI), Italy
}

To cite this article: Ghiani A, Aina R, Asero R, Bellotto E, Citterio S. Ragweed pollen collected along high-traffic roads shows a higher allergenicity than pollen sampled in vegetated areas. Allergy 2012; DOI:10.1111/j.1398-9995.2012.02846.x.

\section{Keywords \\ Ambrosia artemisiifolia; ragweed pollen allergens; sub-pollen particles; traffic-related pollutants. \\ Correspondence \\ Sandra Citterio, Dipartimento di Science Ambientali, Università di Milano-Bicocca, Piazza della Scienza 1, 20126 Milano. \\ Tel.: +390264482934 \\ Fax: +3902 64482996 \\ E-mail: sandra.citterio@unimib.it \\ *These authors contributed equally to this work.}

Accepted for publication 14 April 2012

DOI:10.1111/j.1398-9995.2012.02846.x

Edited by: Reto Crameri

\begin{abstract}
Background: Pollutants may affect pollen allergenicity and thus the prevalence of allergies. Although a few studies are available in literature, the connection between pollution and the allergenic potential of pollen has yet to be clearly defined. The objective of this study was to evaluate the effect of traffic-related pollution on the allergenicity of ragweed (Ambrosia artemisiifolia L.) pollen through a field-based experiment.

Methods: Mature pollen grains were collected from ragweed plants grown along main roadsides and in vegetated areas of Po river plain. The percentage of subpollen particle-releasing grains (SPPGs) was evaluated immediately after sampling by microscope and image analysis. Immunochemistry and LC-MS/MS were applied to assess the whole allergenicity and the allergen pattern characterizing the different pollen samples.

Results: No statistical difference was detected in the percentage of SPPGs among pollen samples. Specifically, after hydration, the mean percentage was very low $(<4 \%)$ in all the samples, regardless of the site of origin. On the contrary, pollen collected along high-traffic roads showed a higher whole allergenicity than pollen from low-traffic roads and vegetated areas which showed a reactivity similar to that of the commercial pollen 'Allergon', used as a standard. The detected higher allergenicity levels were attributed to both quantitative and qualitative differences in allergen pattern.

Conclusion: Our findings show that pollen collected at different sites contains different amount and number of allergens and suggest that traffic-related pollution enhances ragweed pollen allergenicity, which may contribute to the increasing prevalence of ragweed allergy in Lombardy plain.
\end{abstract}

Pollutants have been indicated as one of the contributing factors to the increased incidence of allergic diseases observed in recent years. Pollen is a very important source of allergens, and the prevalence of pollinosis, in particular, can be affected by the presence of environmental pollutants. Pollutants can exert their action at different levels: (i) directly upon the individual, by damaging the mucous membranes and thus facilitating the penetration of pollen allergens and their interaction with the cells of the immune system, or (ii) indirectly upon pollen and pollen allergens. Regarding the latter aspect, it has been demonstrated that air and soil contaminants can affect plant growth and reproduction. For instance, it has been observed, in both laboratory and field conditions, that the increased atmospheric concentration of carbon dioxide $\left(\mathrm{CO}_{2}\right)$ and the accompanying increase in temperature have promoted allergenic plant productivity and then allergenic pollen production (1-3). Pollutants were also demonstrated to interact directly with pollen grains, affecting cell wall structure and leading to increased release of allergens or sub-pollen particles containing allergens (4). In addition, it was observed that pollutants can form complexes with pollen grain or allergen-carrying particles released by plants, enhancing the allergic reaction in exposed subjects by acting as adjuvant (5). Finally, some studies showed that plants, grown in heavily polluted 
areas, can express new allergenic proteins or modify the amount of known allergens or their antigenic properties through post-translational modifications (PTMs; 6-11).

Overall, these studies demonstrated a close association between environmental pollution and pollen allergy. However, most of them were laboratory-based and aimed to assess the effects of specific pollutants directly on allergenic proteins or isolated pollen grains, whereas few field investigations have been carried out to compare the allergenicity and the allergen patterns of pollen from plants grown in areas characterized by different degrees of pollution.

In this study, we report the results of a field experiment aiming to assess the effect of traffic-related pollution on ragweed pollen allergens. It was of particular interest to investigate whether ragweed pollen from plants grown along heavytraffic roads is more allergenic than ragweed pollen from plants grown in vegetated areas, where trees and shrubs can ameliorate air quality by absorbing gaseous pollutants.

\section{Materials and methods}

\section{Study area and pollen sampling}

The study was carried our within the plain of the Lombardy Region (northern Italy). Sampling areas were selected on the basis of the results of aerobiological investigations of ragweed pollen counts and on the basis of the road network connecting the principal urban centres of the region. Particular attention was given to the west part of the plain where the prevalence of ragweed allergy is very high $(12,13)$. Figure 1 shows the twelve selected areas. Seven of these areas were located at the sides of heavy-traffic roads (within $10 \mathrm{~m}$ from the traffic source), two were at the side of low-traffic roads (within $10 \mathrm{~m}$ ), and the remaining three were represented by vegetated areas located far (at least $500 \mathrm{~m}$ ) from motor roads and from industrialized and agricultural lands. Vegetated areas were only three because of the high level of anthropization, density of industries and roads of the Po river plain. Mature pollen grains, naturally released from anthers, were collected from ragweed plants during 2010 summer (July-September). Samples were also collected during 2011 season to confirm 2010 results. Pollen samples were transported to the laboratory where they were immediately analysed to assess the percentage of pollen grains releasing sub-pollen particles $(\varnothing<4-5 \mu \mathrm{m}$; SPPGs) and processed to obtain protein extracts. Detailed information regarding pollen sampling was reported in Data S1 (online Supporting information). Climate variables (temperature and relative humidity) were measured for each selected area during pollen sampling and reported in Table S1 (online Supporting information). The concentrations

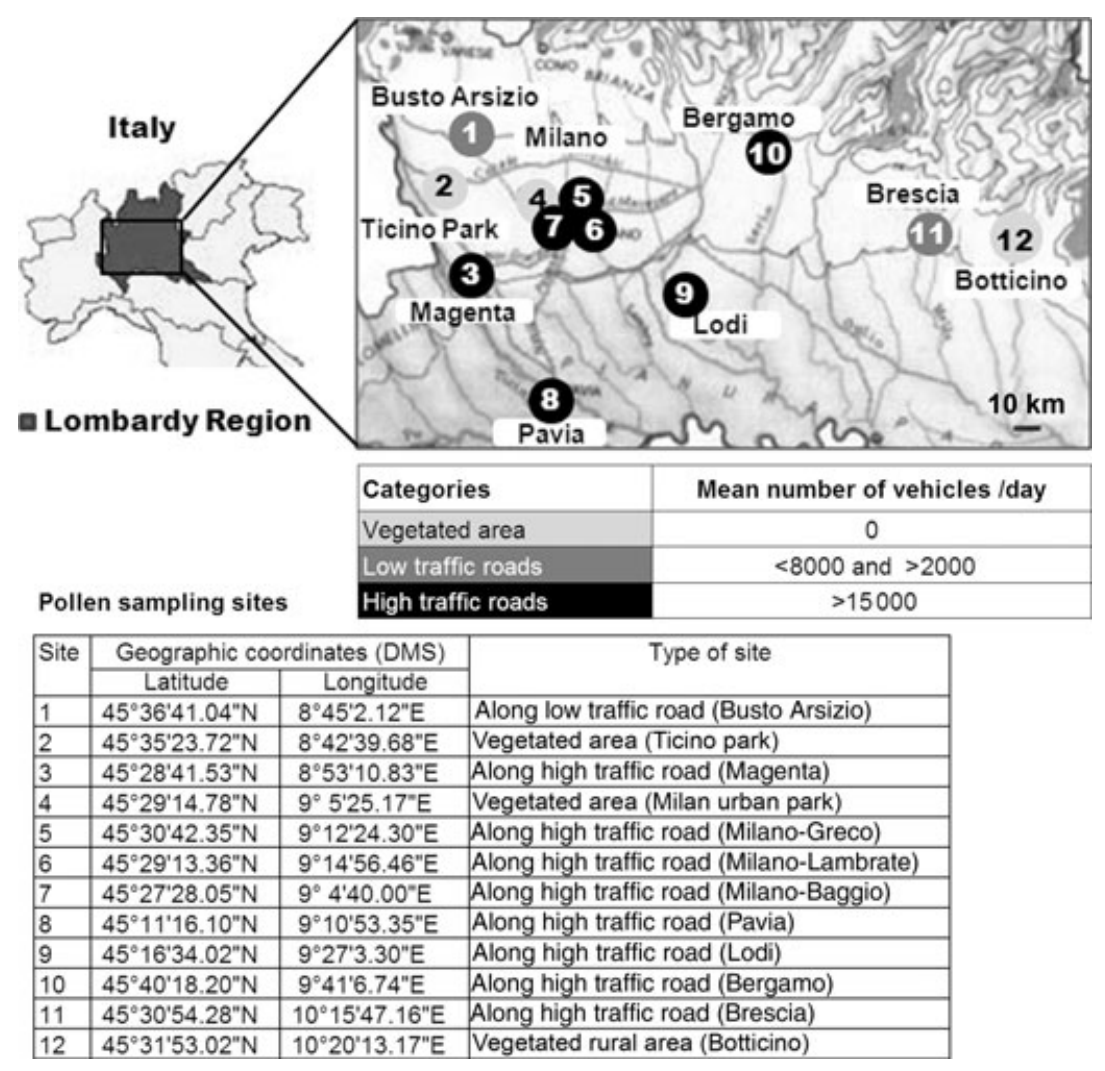

Figure 1 Map of the study area (Lombardy Po river plain) showing the 12 sites in which pollen was collected. The sites were classified in three categories on the basis of the number of motor vehicles travelling on road per day. Light grey: vegetated sampling sites; dark grey: sampling sites along low-traffic roads; black: sampling sites along high-traffic roads. Geographic coordinates (DMS) are reported for each site. 
of the main pollutants related to the period of ragweed growth and reproduction were also assessed (Fig. S1).

\section{Measurement procedures}

All the following measurements are described in detail in Data $\mathrm{S} 1$.

\section{Sub-pollen particles release analysis}

The percentage of SPPGs was estimated by means of a Zeiss Axioplan light microscope connected to a Leica DC 300F camera for photo documentation. The acquired digital images were analysed with the Image-Pro plus program (Media Cybernetics, Silver Spring, MD, USA).

Patients and preparation of a sera mix

Sera from 12 adult subjects were selected for their ability to specifically detect ragweed allergens and were pooled to carry out all immunochemical analyses. The serum pool was aliquoted and stored at $-20^{\circ} \mathrm{C}$ until use.

\section{Preparation of pollen protein extracts}

Debries (i.e. small pieces of insects, small stones, etc.), which were frequently mixed to the collected pollen, were removed under a binocular stereo microscope to obtain samples made of pollen grains only.

Soluble protein extracts were prepared according to Aina et al. (11). At least five independent extracts were prepared for each sampling area. Protein extracts were used for protein slot blot and 1D immunoblot analyses.

\section{Protein slot blot}

Slot blot technique was applied to assess the whole allergenicity of pollen collected at different sites. Equal volumes of protein extracts were bound to nitrocellulose membrane and first stained with Ponceau $\mathrm{S}$ staining solution $[0.1 \%(\mathrm{w} / \mathrm{v})$ Ponceau $\mathrm{S}$ in $5 \%(\mathrm{v} / \mathrm{v})$ acetic acid] to assess the amount of proteins loaded in each well. Membranes were then used to evaluate the immunoreactivity of the different pollen extracts to the sera mix from ragweed allergic patients. Image analysis was applied to quantify reactivity signals. Five independent experiments including at least three different samples for each site were performed.

\section{D SDS-PAGE and immunoblotting}

Pollen extracts $(30 \mu \mathrm{l} /$ lane $)$ were separated by $14 \%$ SDSpolyacrylamide gels according to Laemmli (14). Gels were either stained with colloidal Coomassie Blue G-250 (0.1\% Coomassie Blue G250, $170 \mathrm{~g} / 1$ ammonium sulphate, 34\% methanol, $3 \%$ phosphoric acid) or transferred to nitrocellulose membrane. Immunoblotting was performed according to Aina et al. (11). Nitrocellulose filter saturation and seramix reaction were performed as reported for slot blot membranes.

\section{LC-MS/MS and IgE-binding proteins identification}

Immunoreactive bands were carefully excised from Coomassie-stained 1D gels and subjected to in-gel trypsin digestion according to Aina et al. (11). The tryptic fragments were analysed by LC-MS/MS. Protein identity was searched after peptide sequence attributions by using Global Proteome Machine (GPM) software (www.thegpm.org) against available plant databases. Peptide sequences that were not identified through this database search method were further analysed for a de novo peptide sequencing with the PepNovo Sequencing Algorithm (15). All peptides were also analysed using MS-BLAST software (Basic Local Alignment Tool Mass Spectrometry, http://dove.embl-heidelberg.de/Blast2/msblast.html) to obtain the putative protein identity.

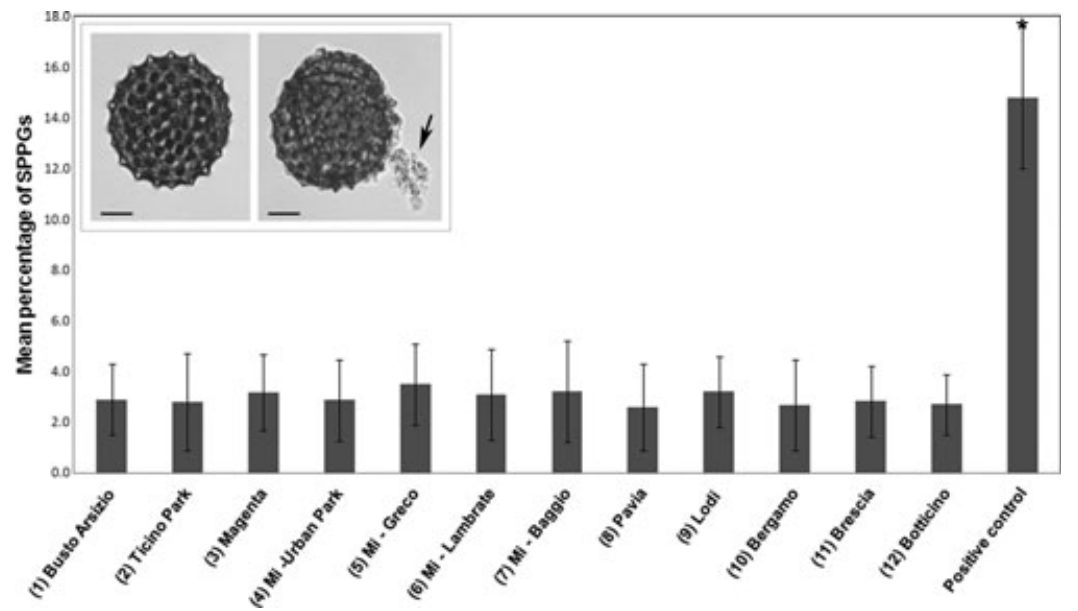

Figure 2 Mean percentage ( \pm standard deviation) of SPP-releasing pollen grains (SPPGs) in samples from the single sampling sites, measured after 15 min of hydration. For each site, the percentage was assessed in at least five samples. (*) Statistically different
$(P<0.01)$. Positive control: Poa pratensis pollen from plants grown in cadmium-contaminated soil. Inset: intact and SPPG after water contact. SPPS are indicated by arrow. Scale bar: $5 \mu \mathrm{m}$. 


\section{Statistics}

Data were analysed by GRAPHPAD PRISM program for Windows applying ANOvA or Kruskal-Wallis nonparametric procedures.

\section{Results}

\section{SPPG analysis}

Pollen grains collected at different sites were immediately analysed for their propensity to release sub-pollen particles. The percentage of sub-pollen particle-releasing grains (SPPGs) was evaluated by light microscopy $15 \mathrm{~min}$ after the contact of pollen with water. In Fig. 2 (inset), an example of a ragweed SPPG is shown. On average, the percentage of SPPGs was very low, $<4 \%$ in all the pollen samples, regardless of the site of origin (Fig. 2). No statistical difference was detected among the samples, given the high data variability. The percentage of SPPGs was also assessed after 30 and 60 min of hydration, with results similar to those obtained from the analysis performed 15 min after the contact of pollen with water.
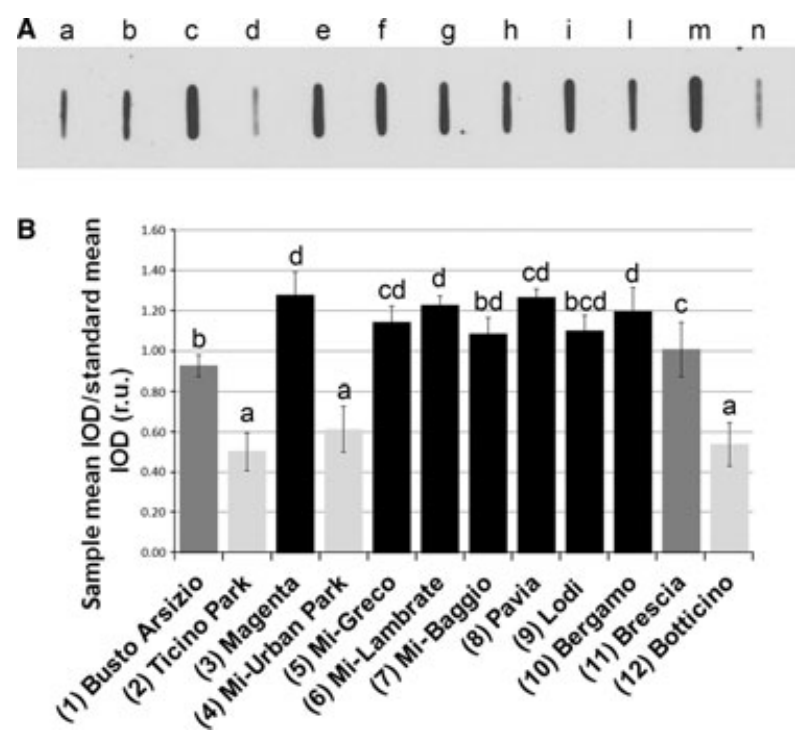

Figure 3 Total allergenicity of field pollen samples assessed with slot blotting. (A) Representative slot blot membrane probed with a pool of selected patient sera; (a) Standard (Allergon); (b) Busto Arsizio; (c) Magenta; (d) Ticino; (e) Mi-Lambrate; (f) Mi-Baggio; (g) Pavia; (h) Bergamo (i) Lodi; (I) Brescia; (m) Mi-Greco; (n) Mi-Urban Park. (B) Assessment of total pollen allergenicity through image analysis: the integrated optical density (IOD) of immunoreactive spots with respect to the IOD of the standard (field sample IOD/standard IOD) was measured. The results reported are the mean of five independent experiments. Different letters indicate significant differences among the samples (ANOvA and Duncan test, $P \leq 0.05$ ). Light grey bars, vegetated sampling sites; dark grey bars, sampling sites along low-traffic roads; black bars, sampling sites along high-traffic roads; r.u., relative units.

\section{Assessment of pollen allergenicity}

To assess the total allergenicity of pollen samples, protein slot blot analysis was carried out. Slot blot technique was used to preserve protein conformation, on which $\operatorname{IgE}$ binding may depend. Identical and comparable volumes of soluble protein extracts were bound on a nitrocellulose membrane and subjected to immunoreaction with a sera mix from selected ragweed allergic patients. Figure 3A shows a representative membrane after immunodetection. Image analysis was applied to quantify immunochemical signals: the integrated optical density (IOD) of immunoreactive spots with respect to the IOD of standard (field sample IOD/standard IOD) was measured. The mean results of five independent experiments were calculated and statistically analysed (Fig. 3B). On average, all the pollen samples collected along heavy-traffic roads (black bars) showed a statistically higher IgE-binding signal, ranging from 1.05 to 1.27 , than pollen sampled in vegetated areas (light grey bars), whose immunochemical signal ranged from 0.55 to $0.63(P<0.05)$. Pollen samples collected along low-traffic roads (dark grey bars) showed reactivity signals ranging from 0.93 to 0.96 , which were statistically higher than those measured for pollen sampled in vegetated areas and lower, although not statistically different, than those calculated for samples collected along heavy-traffic roads $(P<0.05)$.

To investigate the cause of the difference in slot blot allergenicity signals, 1D immunoblot, probed with the same sera mix used for slot blotting, was carried out. Figure 4A shows a representative 1D immunoblot picture, where the single allergens, identified by LC-MS/MS, can be clearly observed.

Image analysis performed on 1D immunoblots from three independent experiments confirmed the slot blot results indicating a statistically significant lower allergenicity of pollen collected in vegetated areas in comparison with that of pollen collected along traffic roads (Fig. 4B). The diverse allergenicity was ascribed to differences in allergen pattern, which, in all experiments, was similar among pollen samples collected in the same area but different among pollen samples collected at different sites. As shown by image analysis (Fig. 4C), the differences were both qualitative and quantitative, that is, related to both the number and the amount/allergenic potential of allergens. The major ragweed allergen, Amb a 1, was expressed in all the pollens, but its IgE-binding reaction was lower in the samples from vegetated sites $(P<0.05)$. The allergenic reaction of pollens from vegetated areas was totally ascribed to $\mathrm{Amb}$ a 1 isoforms; this pollen did not contain detectable concentrations of additional allergens, such as Amb a 6 and Amb a 10 , which instead contributed to the allergenicity of most of the other samples. Specifically, Amb a 6 was detected in pollen from four of the seven heavy-traffic sites and from both lowtraffic areas. In contrast, Amb a 10 was detected only in pollen from five of the seven high-traffic sites. Low amount of both Amb a 6 and Amb a 10 was also detected in standard pollen (commercial pollen from Allergon company). In addition, a globulin-like protein, reacting with patient sera mix, was identified in all pollen samples collected along high-traffic roads 


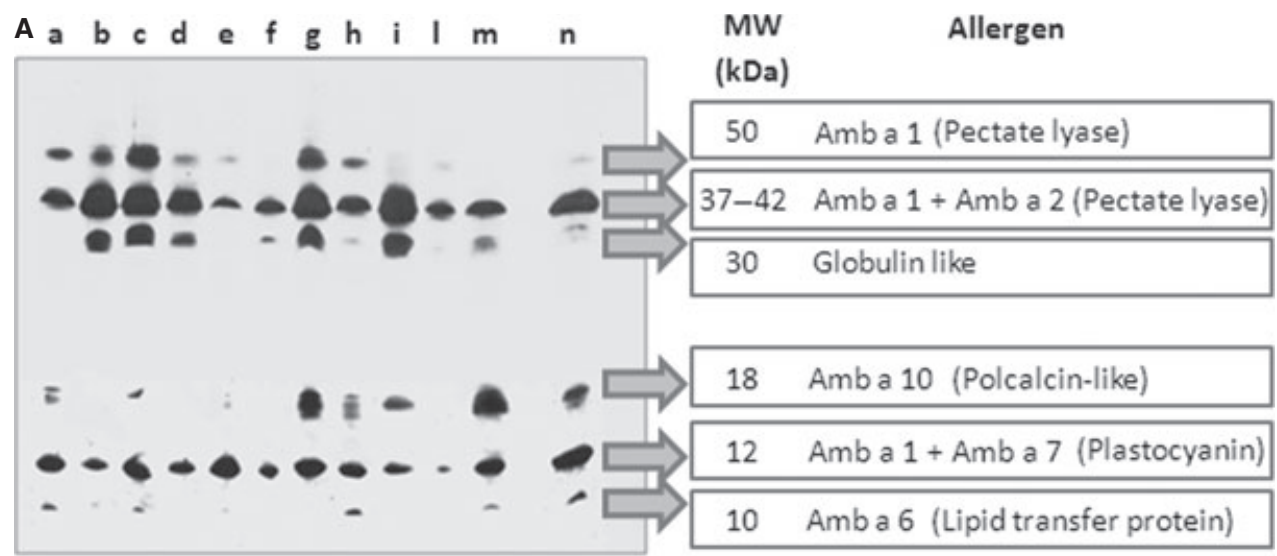

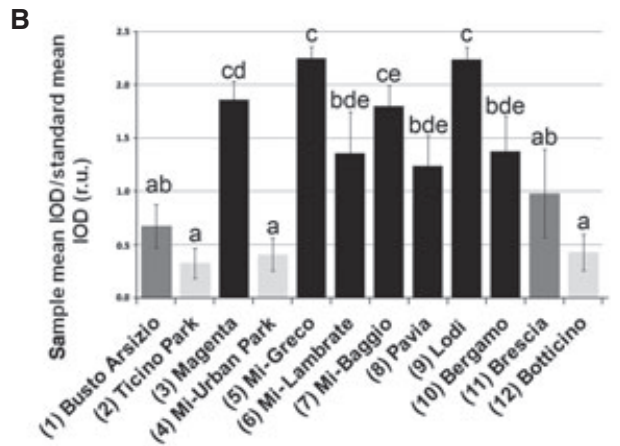

Figure 4 Allergen detection in ragweed pollen extracts from samples collected at different sites. (A) Representative immunoblot probed with the same pool of selected patient sera used for slot blot and the corresponding protein identification by LC-MS/MS. (a) Standard (Allergon); (b) Mi-Baggio; (c) Lodi; (d) Bergamo; (e) Brescia; (f) Busto Arsizio; (g) Mi-Greco; (h) Pavia; (i) Magenta; (l) MiUrban Park; (m) Mi-Lambrate; (n) Mi-Baggio. (B) Assessment of total pollen allergenicity through image analysis: the integrated optical density (IOD) of immunoreactive bands with respect to the IOD

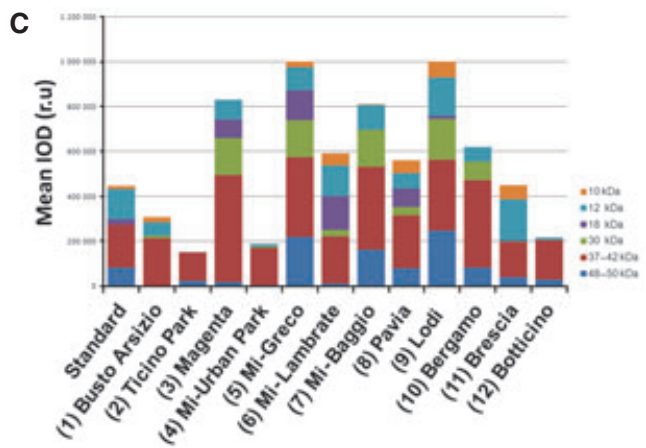

of the standard correspondent bands (sample IOD/standard IOD) was measured. The sum of the mean IOD of the single bands for each sampling site with respect to the standard was reported in the graphic. Light grey bars: vegetated sampling sites; dark grey bars: sampling sites along low-traffic roads; black bars: sampling sites along high-traffic roads. (C) Contribution of the single allergens to the total allergenicity of pollen for each site. The mean IOD of the single bands for each sampling site and for the standard was reported in the graphic; r.u., relative units.
SLOT BLOT

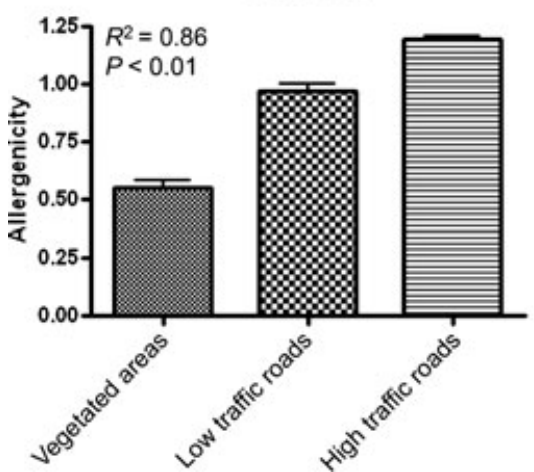

Figure 5 Statistical correlation between allergenicity and the sampling area type. ANOVA and post-test for linear trend were applied.
1D-IMMUNOBLOT

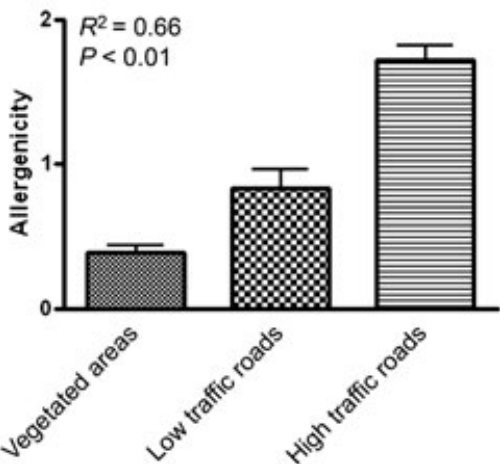

The linear trend resulted significant $(P<0.01)$ for both slot blot and $1 \mathrm{D}$ immunoblot data. 
and was more abundant in higher allergenic pollens (Fig. 4C). Interestingly, a low amount of this protein was detected in pollen samples from one of the two low-traffic sites and was absent in vegetated site samples and in standard pollen.

On the whole, as clearly visualized in Fig. 5, higher allergenic pollen was collected along heavy-traffic roads that contained a higher number and amount of allergens than pollen sampled in vegetated areas that resulted less allergenic. These results obtained for 2010 pollen samples were confirmed by the slot blot analysis of few representative samples collected during 2011 along the same high-traffic roads and in vegetated areas (Fig. S2).

\section{Discussion}

Ambrosia artemisiifolia L. (short ragweed) is becoming the principal cause of pollinosis in Lombardy. It is an alien and invasive plant native to eastern and central North America, but now persistently spreading in Europe. The ability of this plant to produce enormous amounts of pollen could explain the rapid development of ragweed sensitization rates registered in Lombardy $(12,13)$, although it is likely that many other factors, such as environmental pollutants, may contribute to this phenomenon. In general, pollutants are able to exacerbate the allergic reaction by acting upon the individual and/or affecting pollen structure and composition. In this work, we designed an outdoor experiment to realistically address the impact of motor vehicle-related pollution on ragweed pollen. A field approach was chosen because, although many useful laboratory-based studies have been performed to investigate the effect of air contaminants on pollen, the applicability of their results to in situ conditions remained indicative as the treatment with pollutants was applied only to dehydrated mature pollen and not to the growing plant. In addition, only single contaminants were usually tested, not taking into account synergistic and antagonistic effects of pollutants. In our study, by comparing the allergenicity of pollen collected from plants grown along high- and low-traffic roads and in vegetated areas, we showed a different allergen-IgE reactivity of pollens sampled in the three environments. Specifically, pollen collected along high-traffic roads during 2010 and 2011 seasons showed a higher allergenicity because of a higher allergen-IgE reactivity. These findings are in keeping with the findings by Cortegano et al. (8) and Suárez-Cervera et al. (16), which showed that Cupressus arizonica pollen collected in close proximity to roads contained higher amounts of the allergen Cup 3. Other previous field-based experiments, which assessed the differences between pollen from rural and urban areas, provided divergent results. For instance, the amount of Lol p 5 in Lolium pollen was higher in urban areas (17), whereas Phl p 5 allergen was more abundant in pollen collected from rural areas (18), and the amount of Bet v 1 was similar in the two environments (19). In our opinion, the discrepancy among these results should be ascribed to the heterogeneity of the urban areas. Interestingly, in our study, ragweed plants collected inside a Milan-urban park separated from traffic streets by a high and dense vegetation showed a very low allergenic potential in comparison with pollen sampled from plants grown in Milan, but along high-traffic roads. A recent study of Yin et al. (20) reported that vegetation in urban parks may remove large amounts of airborne pollutants. The authors demonstrated by a field experiment that pollutant concentrations decrease from roadside to the interior of parks and that urban trees and shrubs can ameliorate air quality by absorbing gaseous pollutant and trapping particulates. The presence of vegetation, forming a barrier to traffic pollutants, could thus explain the low allergenicity of the pollen collected inside the Milan-urban park and partially explain the contrasting data about the difference in allergen content of pollen from urban and rural areas that are present in literature. The importance of the homogeneity of sampling sites is therefore evident, and it is also clear that the distance between plants and the source of traffic pollutants is an additional important factor that should be considered when the effect of traffic-related pollution is evaluated. Ragweed plants prefer roadsides and produce pollen in organs that are very close to vehicle exhaust pipes. This is a favourable condition to investigate the direct effect of traffic-related pollutants on pollen allergenicity. In our study, traffic pollution affected quantitatively and qualitatively the allergen pattern of ragweed pollen. Specifically, the higher allergenicity of pollen from plants grown along high-traffic road compared to pollen from vegetated areas was due both to a higher IgE reactivity of the major allergens Amb a 1 and Amb a 2 and to the expression of minor allergens, such as Amb a 6 and Amb a 10. In addition, a globulin-like allergen contributed to the detected higher allergenicity. This last protein belongs to the cupin superfamily, which contains many major plant food allergens (21). Proteins homologous to these food allergens were also found in plant pollens (22), although their allergenic potential was never tested and their function never defined. Concerning the higher IgE reactivity of Amb a 1 and Amb a 2 detected in pollen from high-traffic roads, it was related to a higher amount of these proteins, as observed on 1D gels stained with colloidal Coomassie Blue. However, we cannot exclude that conformational changes owing to post-translational modifications have occurred, modifying allergen potential. In fact, nitrogen dioxide, the major trafficrelated pollutant, enhances the allergenicity of birch pollen allergen Bet $\mathrm{v} 1$ through the formation of nitrotyrosine residues (10). Unfortunately, in this regard, our analysis with anti-nitrotyrosine antibody did not provide any conclusive result (data not shown). In addition, we cannot exclude the influence of climatic conditions, as some papers report a different release of allergens from the same plant species grown in different climate (23). Nevertheless, in our experiment, the variation in temperature and humidity was not correlated to allergenicity, maybe because the study area was restricted to Lombardy Po plain, which has a continental climate quite uniform. Moreover, genetic diversity among sampled populations could have contributed to the different allergenicity, although on the basis of the literature $(24,25)$ ragweed populations do not statistically differ in terms of diversity and pollen was collected from many individual plants for each population. 
Traffic pollution can also modify the cell wall structure facilitating the release of sub-pollen particles (SPPs) that contain allergens. This effect was observed in Phleum pollen treated with $\mathrm{NO}_{2}$ and $\mathrm{O}_{3}(4)$ and in pollen of Poa annua plants grown in cadmium-contaminated soil (11). Ragweed pollen was also demonstrated to release SPPs after hydration (26). Nevertheless in our experiment, the percentage of SPP-releasing grains was very low $(<4 \%)$ and was not significantly higher in pollen samples collected along high-traffic roads than in those collected in vegetated areas. The different behaviour between grass and ragweed pollens is likely due to the difference in cell wall structure that is slight and thick, respectively. Thus, our results suggest that the effect of traffic-related pollution on SPP release is species specific, and we cannot exclude that this is the case also for allergen expression and reactivity that could depend on the plant sensitivity to the single pollutants and on the reproductive plant organ and pollen structure. Nonetheless, it is of great relevance that, at least for ragweed, the integrated action of trafficrelated pollutants seems able to enhance pollen allergenicity, thus contributing to the rapid increase in sensitization rate.

\section{Acknowledgments}

The authors wish to thank Professor Pietro Fumagalli for statistical analyses and Dr Rodolfo Gentili and Dr Federica Gilardelli for their help in pollen sampling.

\section{Author contributions}

Drs Ghiani, Aina and Bellotto performed sampling and laboratory experiments. Dr Asero collected patient sera and actively participated to the manuscript writing. Dr Citterio conceived the idea and wrote the manuscript. All the authors carried out data analysis and interpretation.

\section{Funding}

This work was supported by the Italian Ministry of University and Research, by Lombardy Region (Italy) and by a grant from Fondazione Banca del Monte di Lombardia.

\section{Conflict of interest}

None of the authors have any conflict of interests to disclose regarding this manuscript.

\section{Supporting Information}

Additional Supporting Information may be found in the online version of this article:

Data S1. Materials and methods.

Figure S1. Main air pollutant concentrations recorded in the sampling sites during the plant growth and reproduction period (April-September).

Figure S2. Allergenicity of pollen samples collected in vegetated areas and along high traffic roads during 2011 season (from mid-July to mid-September).

Table S1. Mean temperature $\left({ }^{\circ} \mathrm{C}\right)$ and relative humidity in the sampling sites during 2010 pollen sampling season (from mid-July to mid-September).

Please note: Wiley-Blackwell are not responsible for the content or functionality of any supporting materials supplied by the authors. Any queries (other than missing material) should be directed to the corresponding author for the article.

\section{References}

1. Wayne P, Foster S, Connolly J, Bazzaz F, Epstein P. Production of allergenic pollen by ragweed (Ambrosia artemisiifolia L.) is increased in $\mathrm{CO}_{2}$-enriched atmospheres. Ann Allergy Asthma Immunol 2002;88: 279-282.

2. Ziska LH, Gebhard DE, Frenz DA, Faulkner S, Singer BD, Straka JG. Cities as harbingers of climate change: common ragweed, urbanization, and public health. J Allergy Clin Immunol 2003;111:290-295.

3. Rogers CA, Wayne PM, Macklin EA, Mullenberg ML, Wagner CJ, Epstein PR et al. Interaction of the onset of spring and elevated atmospheric $\mathrm{CO}_{2}$ on ragweed (Ambrosia artemisiifolia L.) pollen production. Environ Health Perspect 2006;114:865-869.

4. Motta AC, Marlierec M, Peltred G, Sterenbergb PA, Lacroixc G. Traffic-related air pollutants induce the release of allergen-containing cytoplasmic granules from grass pol- len. Int Arch Allergy Immunol 2006;139:294 298.

5. Cecchi L, D'Amato G, Ayres JG, Galan C, Forastiere F, Forsberg B et al. Projections of the effects of climate change on allergic asthma: the contribution of aerobiology. Allergy 2010;65:1073-1081.

6. Masuch G, Franz JTH, Schoene K, Müsken $\mathrm{H}$, Bergmann $\mathrm{KCH}$. Ozone increases group 5 allergen content of Lolium perenne. Allergy 1997;52:874-875.

7. Singer BD, Ziska LH, Frenz DA, Gebhard DE, Straka JG. Increasing Amb a 1 content in common ragweed (Ambrosia artemisiifolia) pollen as a function of rising atmospheric $\mathrm{CO}_{2}$ concentration. Funct Plant Biol 2005;32:667-670.

8. Cortegano I, Civantos E, Aceituno E, del Moral A, López E, Lombardero $\mathrm{M}$ et al. Cloning and expression of a major allergen from Cupressus arizonica pollen, Cup a 3, a
PR-5 protein expressed under polluted environment. Allergy 2004;59:485-490.

9. Petersen A, Schramm G, Schlaak M, Becker WM. Post-translational modifications influence $\operatorname{IgE}$ reactivity to the major allergen $\mathrm{Phl}$ p 1 of timothy grass pollen. Clin Exp Allergy 1998;28:315-321.

10. Gruijthuijsen YK, Grieshuber I, Stöcklinger A, Tischler U, Fehrenbach T, Weller MG et al. Nitration enhances the allergenic potential of proteins. Int Arch Allergy Immunol 2006;141:265-275.

11. Aina R, Asero R, Ghiani A, Marconi GM, Albertini E, Citterio S. Exposure to cadmium-contaminated soils increases allergenicity of Poa annua L. pollen. Allergy 2010;65:1313-1321.

12. Asero R. Birch and ragweed pollinosis north of Milan: a model to investigate the effects of exposure to "new" airborne allergens. Allergy 2002;57:1063-1066. 
13. Tosi A, Wüthrich B, Bonini M, PietragallaKöhler B. Time lag between Ambrosia sensitisation and Ambrosia allergy A 20-year study (1989-2008) in Legnano, northern Italy. Swiss Med Wkly 2011;141:w13253.

14. Laemmli UK. Cleavage of structural proteins during the assembly of the head of bacteriophage T4. Nature 1970;227:680-685.

15. Frank A, Pevzner P. PepNovo: de novo peptide sequencing via probabilistic network modeling. Anal Chem 2005;77: 964-973.

16. Suárez-Cervera M, Castells T, Vega-Maray A, Civantos E, del Pozo V, Fernández-González D et al. Effects of air pollution on Cup a 3 allergen in Cupressus arizonica pollen grains. Ann Allergy Asthma Immunol 2008;101:57-66.

17. Armentia A, Lombardero M, Callejo A, Barber D, Martin Gil FJ, Martin-Santos JM et al. Is Lolium pollen from an urban environment more allergenic than rural pollen? Allergol Immunopathol (Madr) 2002;30:218-224.
18. Behrendt H, Tomczok J, Sliwa-Tomczok W, Kasche A, Ebner von Eschenbach C, Becker $\mathrm{WM}$ et al. Timothy grass (Phleum pratense L.) pollen as allergen carriers and initiators of an allergic response. Int Arch Allergy Immunol 1999;118:414-418.

19. Bryce M, Drews O, Schenk MF, Menzel A, Estrella N, Weichenmeier I et al. Impact of urbanization on the proteome of birch pollen and Its chemotactic activity on human granulocytes. Int Arch Allergy Immunol 2010;151:46-55.

20. Yin S, Shen Z, Zhou P, Zou X, Che S, Wang W. Quantifying air pollution attenuation within urban parks: an experimental approach in Shanghai, China. Environ Pollut 2011;159:2155-2163.

21. Mills EN, Jenkins J, Marigheto N, Belton PS, Gunning AP, Morris VJ. Allergens of the cupin superfamily. Biochem Soc Trans 2002;30:925-929.

22. Holmes-Davis R, Tanaka CK, Vensel WH, Hurkman WJ, McCormick S. Proteome mapping of mature pollen of Arabidopsis thaliana. Proteomics 2005;5:4864-4884.

23. Buters JT, Kasche A, Weichenmeier I, Schober W, Klaus S, Traidl-Hoffmann $\mathrm{C}$ et al. Year-to-year variation in release of Bet $\mathrm{v} 1$ allergen from birch pollen: evidence for geographical differences between West and South Germany. Int Arch Allergy Immunol 2008;145:122-130.

24. Gaudeul M, Giraud T, Kiss L, Shykoff JA. Nuclear and chloroplast microsatellites show multiple introductions in the worldwide invasion history of common ragweed, Ambrosia artemisiifolia. PLoS ONE 2011;6: e17658.

25. Chun YJ, LE Corre V, Bretagnolle F. Adaptive divergence for a fitness-related trait among invasive Ambrosia artemisiifolia populations in France. Mol Ecol 2011;20:1378-1388.

26. Bacsi A, Dharajiya N, Choudhury BK, Sur S, Boldogh I. Subpollen particles: carriers of allergenic proteins and oxidases. $J$ Allergy Clin Immunol 2006;118:844-850. 